\title{
DESIGN IMPLEMENTATION AND HARDWARE STRUCTURE FOR IMAGE ENHANCEMENT AND SURFACE ROUGHNESS WITH FEATURE EXTRACTION USING DISCRETE WAVELET TRANSFORM
}

\author{
${ }^{1}$ Syed Jahangir Badashah and ${ }^{2} \mathbf{P}$. Subbaiah \\ ${ }^{1}$ Department of Electronics and Communication, Research Scholar of Sathyabama University, Chennai, India \\ ${ }^{2}$ Department of Electronics and Communication, \\ Dhanalakshmi College of Engineering, Anna University, Chennai, India
}

Received 2013-08-20; Revised 2013-10-04; Accepted 2013-11-20

\begin{abstract}
In this study we provide the implementation design and hardware structure for the architecture proposed in the previous paper "Image Enhancement and Surface Roughness with Feature Extraction using DWT" IEEE DoI/10.1049/cp.2011.0464. The proposed architecture has been implemented in Microwind and Tanner for power analysis and characteristic study. The result shows very low power consumption than the existing method for a series of IEEE standard test images.
\end{abstract}

Keywords: Surface Roughness, Milling, Grinding, Noisy Filter, Edge Detector, Image Enhancement

\section{INTRODUCTION}

Comparing with real time application, the engineering application needs to provide good quality and performance of materials during production. The main quality of surface roughness can be measured by using two techniques, namely optical and stylus techniques. Radii of the diamond can be checked using stylus but surface roughness (Damodarasamy and Raman, 2003) can be checked by optical techniques which are quite expensive. Now-a-days machine vision is used to evaluate and analyze the microscopic defects in surface of the materials. The other methods of surface roughness study is shown in Fig. 1.

\section{MATERIALS AND METHODS}

Human vision can be replaced with machine vision with capturing, compressing and extraction of image in high speed precision manufacturing areas as a mainstream automation tool (Badashah and Subbaiah, 2011). Machine vision (Luk et al., 1989; Al-Kindi et al., 1992) has the advantage of grasping the images online without accounting for factors like vibrations (advantage), noise, intensity (disadvantage) (Tsai and
Tseng, 1999). There is a need for design of recognition systems with capability to adjust to changing environments automatically. Less complicated, highly flexible and more cost-effective computing architectures are required as compared to the traditional ones.

Two dimensional Fourier Transform and Wavelet Transform are applied to the extracted enhanced images in spatial frequency domain. Fourier transform is used for stationary profiles and for non-stationary profiles Wavelet transform is used. The experimental setup is shown in Fig. 2.

\subsection{Feature Extraction using Wavelet Ransforms}

Fourier Transform and wavelet decomposition techniques (family of orthogonal wavelets) are used to extract the features from the image of the surface under test. The features such as the major peak frequency and the principal component magnitude squared value are extracted using Fourier transform. The energy details of the sub band images (Ramamoorthy and Radhakrishnan, 1993), such as, energy total, energy horizontal, energy vertical and energy diagonal are extracted using the wavelet (Db4) multi resolution decomposition algorithm.

Corresponding Author: Syed Jahangir Badashah, Department of Electronics and Communication, Research Scholar of Sathyabama University, Chennai, India 


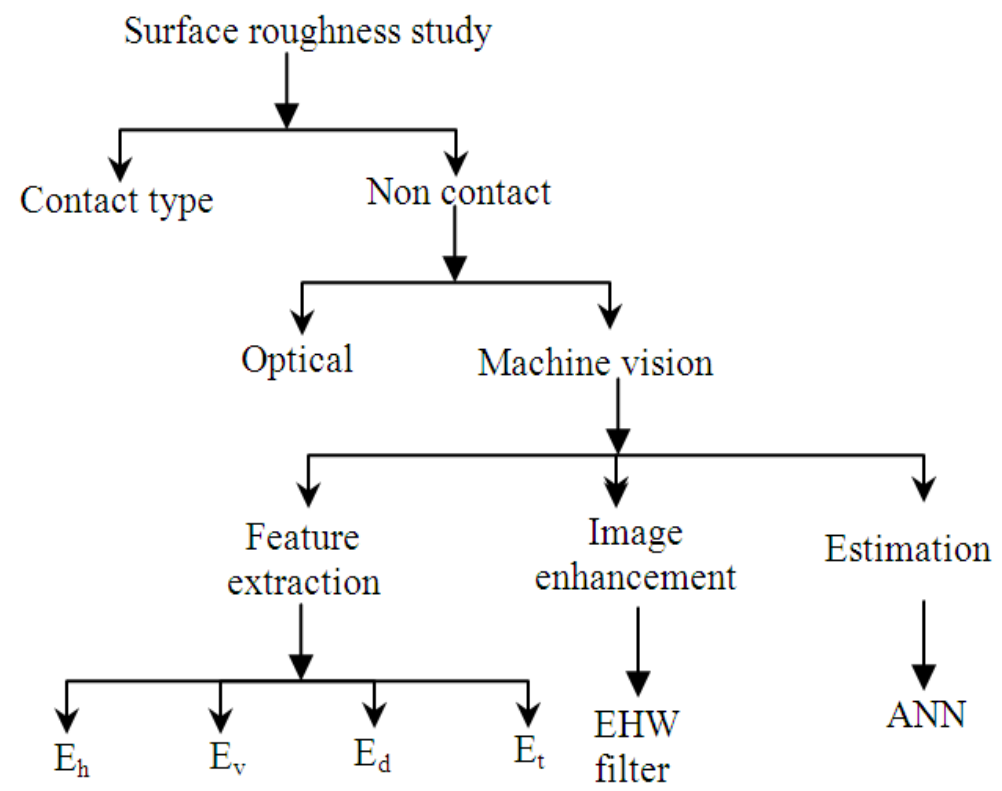

Fig. 1. Surface roughness study

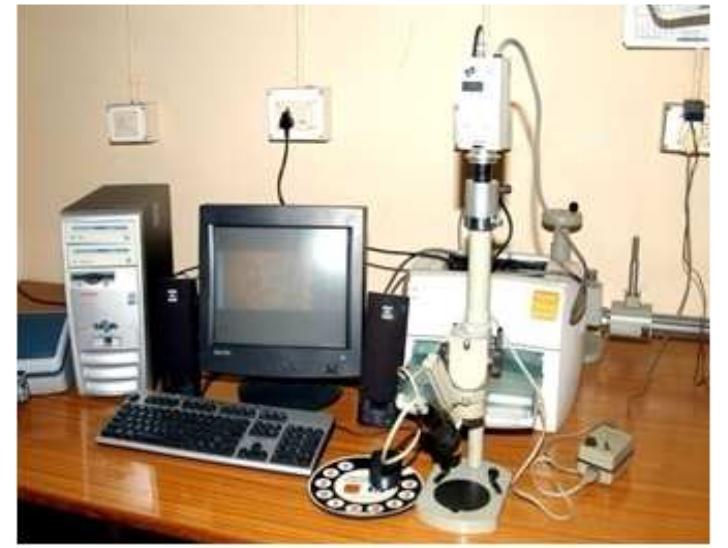

Fig. 2. Experimental setup (CVRDE)

\subsection{Image Enhancement using EHW Filter}

Image degradation occurs due to noise delivered from unavoidable source. The most common noise is impulse noise (Kartik et al., 1997; Cheikh et al., 1998; Astola and Kuosmanen, 1997; Dougherty and Astola, 1994). Enhancement of the image can be done using filtering technique. The processing elelment simulation of evolvable hardware for image enchancement is shown in Fig. 3 and 4. Linear filter are very less resistive to impulse noise. Hence the Median filter, a Non Linear filter has been used for enhancement and implemented using Evolvable Hardware.

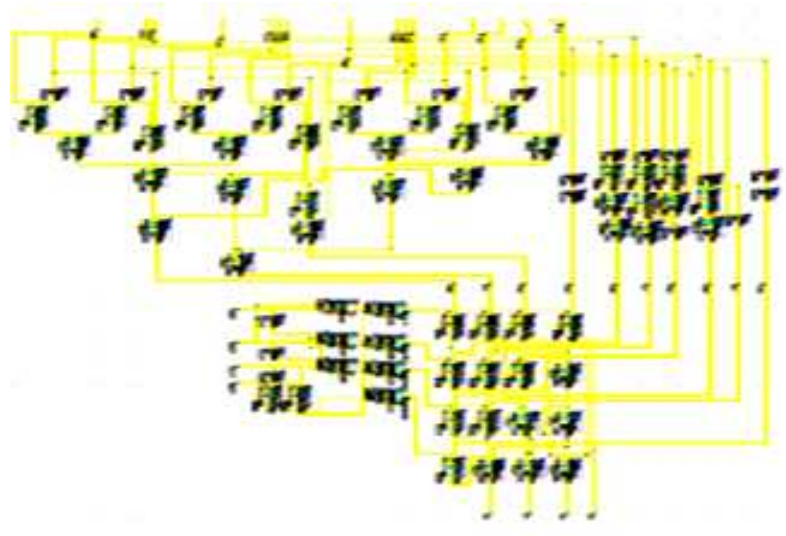

Fig. 3. Multi resolution decomposition using Microwind

\subsection{Surface Roughness Estimation using Neural Network}

From literature survey, regression technique is one of the better methods for surface roughness estimation. But it involves higher complexity and calculation for a little increase of accuracy. As an alternate neural network with back propagation algorithm (Fig. 5) based estimation can be used for surface estimation with higher accuracy and constant resource (Since the topology of the network is constant. Only bias and weights are change with some algorithm). 


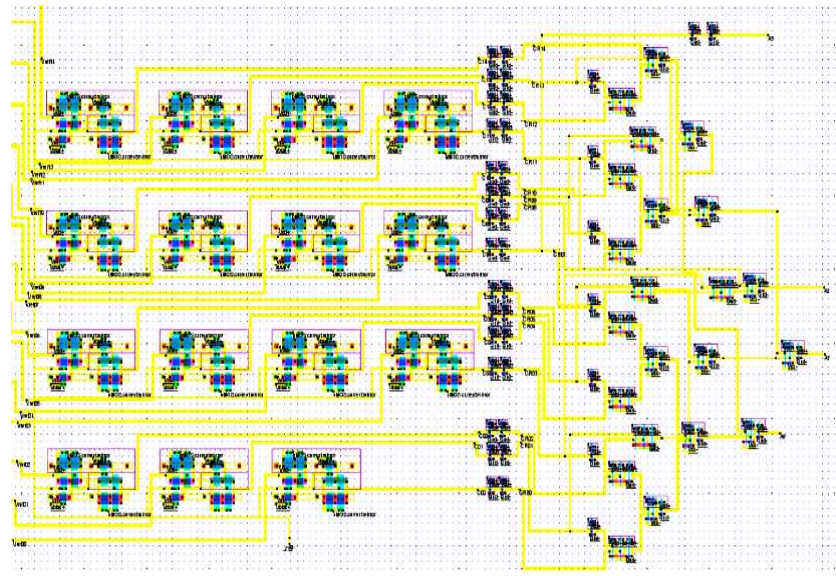

Fig. 4. Processing elements structure of EHW in microwind

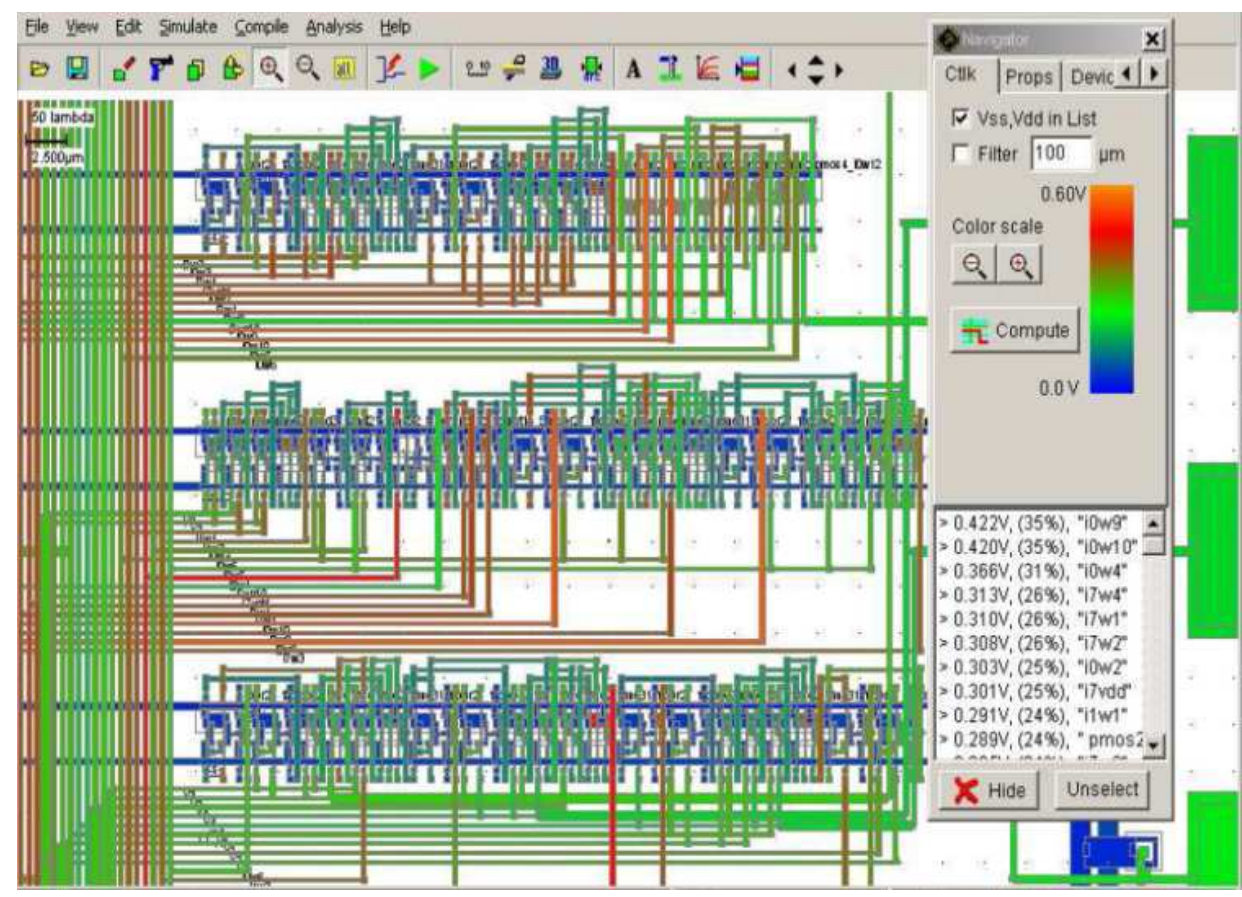

Fig. 5. Neural logic (Hidden Layer neurons and their multiplier) structure using microwind

\subsection{Objectives of this Work}

The major objectives of this research work are:

- In this study, to overcome the problem of high primitive gate level evolvable hardware structure, a function-level evolution (Negoita et al., 2008) is proposed. Domain knowledge is used to select high level computational units that signify directly in the chromosome
- To extract the features of the image using wavelet and fourier transform independently

- Training A Neural Network (ANN) (Samhouri, 2005) and use it for approximating the surface roughness $\mathrm{R}_{\mathrm{t}}$ of industry related components manufactured using processes such as grinding and milling. ANNs have the ability to recognize patterns that are similar, but not identical; it can store information and generalize it. As this would 
introduce huge parallelism, the ANNs exhibit increased computational power that can be used to deal with intricate problems. In this research, backpropagation neural network is used for estimating the surface roughness of the machined surfaces

- A comparison of surface finish attained using proposed scheme with that of using classical and conventional stylus approach

\section{RESULTS AND DISCUSSION}

The resource utilized by the proposed algorithm (Fig. 6) is economical in each and every stage of the proposed algorithm. Though there is trade off at the early stage of the filter design, it remains almost constant and independent of the number of coefficient in the entire implementation of the filter. The minimum number of coefficient will not be of 2 to 6 for a efficient filtering process (Gabbouj, 1996).

The Table 1 and Fig. 6 shows the resource utilized and the Table 2 and Fig. 7 shows the processing time by the existing architecture and the proposed architecture. It is clear from both the cases that the proposed architecture outperforms the existing architecture of DSP implementation. It is also clear from the Fig. 8 and 9 the power requirement is predictable and minimum.

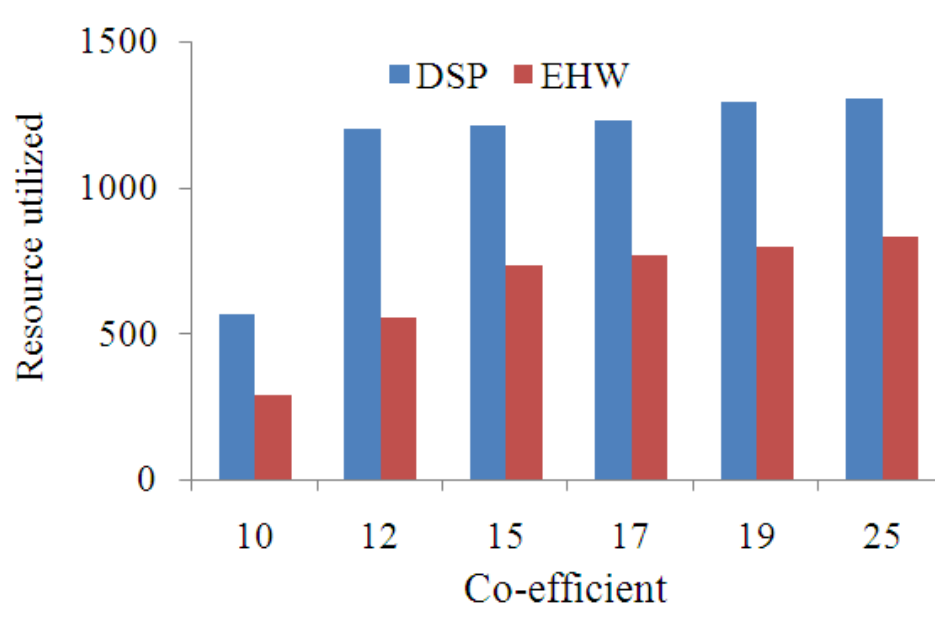

Fig. 6. Comparison of resource utilization of existing DSP based processor and proposed architecture

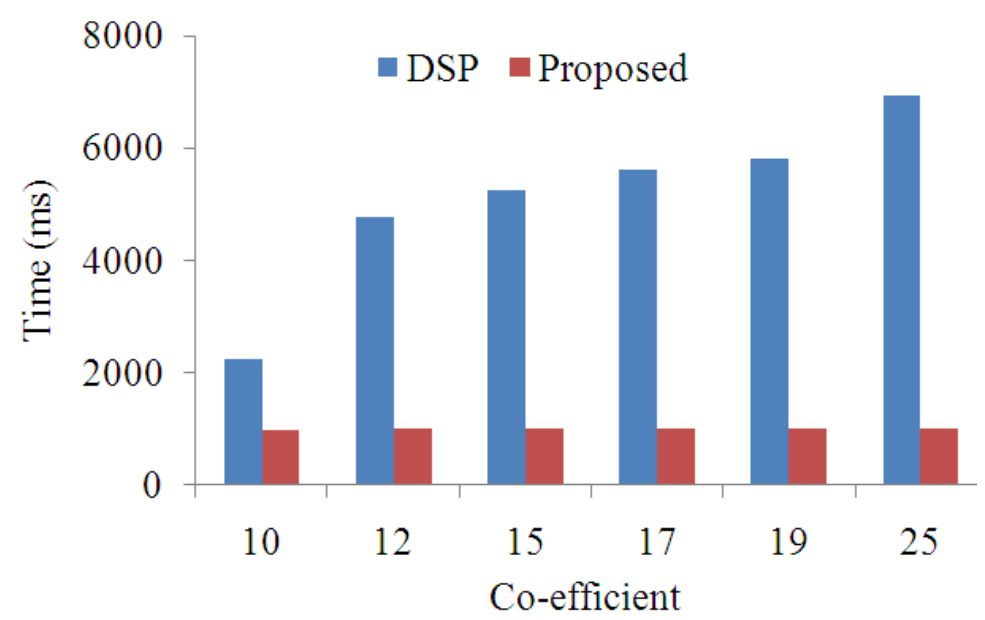

Fig. 7. Comparison of processing time of existing DSP based processor and proposed architecture 
Syed Jahangir Badashah and P. Subbaiah / Journal of Computer Science 10 (2): 347-352, 2014

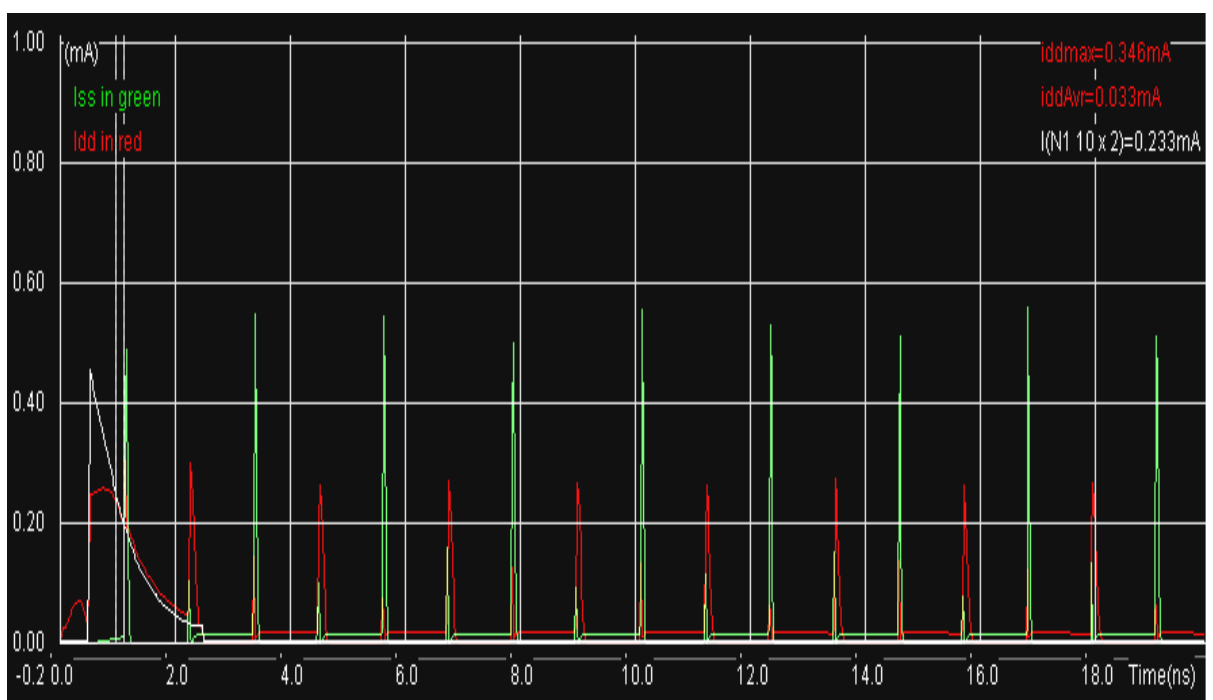

Fig. 8. Power fluctuation of proposed circuit

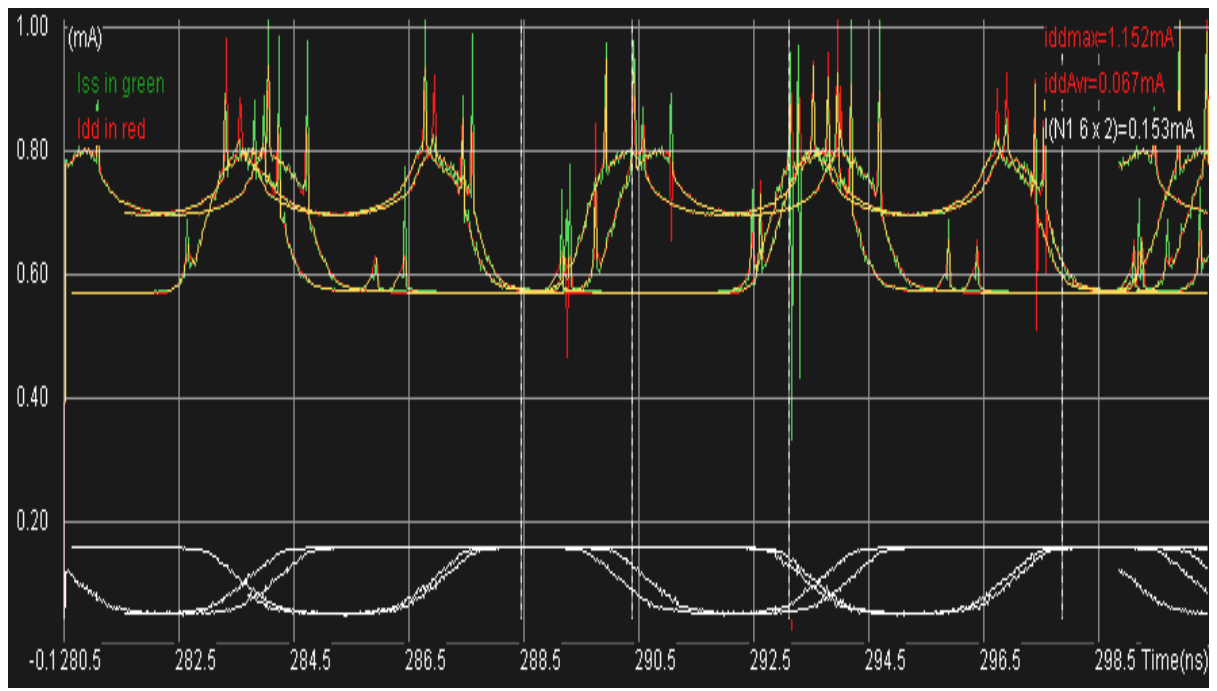

Fig. 9. Power fluctuation of DSP circuit

Table 1. Comparison of resource utilization of existing DSP based processor and proposed architecture

\begin{tabular}{lrl}
\hline Co-eff & DSP & EHW \\
\hline 10 & 563 & 286 \\
12 & 1200 & 551 \\
15 & 1213 & 732 \\
17 & 1226 & 766 \\
19 & 1290 & 799 \\
25 & 1301 & 832 \\
\hline
\end{tabular}

Table 2. Comparison of processing time of existing DSP based processor and proposed architecture

\begin{tabular}{lcl} 
& \multicolumn{3}{c}{ Processing time } \\
& Drocessor and & \\
DSP & Proposed & Speed up\% \\
\hline 2250 & 986 & 228.1 \\
4762 & 999 & 476.6 \\
5263 & 1001 & 561.7 \\
5620 & 1003 & 560.3 \\
5814 & 1004 & 579.0 \\
6944 & 1010 & 687.5 \\
\hline
\end{tabular}




\section{CONCLUSION}

In this study, a vision application capable of performing selective image processing and analysis has been implemented. The filter outperforms conventional designs in terms of performance measure, high speed computation and low power consumption. It is easily scalable and can be mapped with Digital Logic operators with lesser non-linear operation. Hence, the schemes (based on EHW filter, Wavelet and ANN) can successfully replace conventional ones.

\section{REFERENCES}

Al-Kindi, G.A., R.M. Baul and K.F. Gill, 1992. An application of machine vision in the automated inspection of engineering surfaces. Int. J. Product. Res., $\quad 2$ : 241-253. $\quad$ DOI: 10.1080/00207549208942892

Astola, J. and P. Kuosmanen, 1997. Fundamentals of Nonlinear Digital Filtering. 1st Edn., CRC Press, Boca Raton, ISBN-10: 0849325706, pp: 288.

Badashah, S.J. and P. Subbaiah, 2011. Image enhancement and surface roughness with feature extraction using DWT. Proceedings of the 2nd International Conference on Sustainable Energy and Intelligent System, Jul. 20-22, IEEE Xplore Press, Chennai, pp: 754-759. DOI: 10.1049/cp.2011.0464

Cheikh, F.A. L. Khriji, M. Gabbouj and G. Ramponi, 1998. Color image interpolation using vector rational filters. Proceedings of the SPIE Conference on Nonlinear Image Processing, Jan. 24-24, San Jose, CA., pp: 24-30. DOI: $10.1117 / 12.304604$

Damodarasamy, S. and S. Raman, 2003. Texture analysis using computer vision. Comput. Indus., 16: 25-34. DOI: 10.1016/0166-3615(91)90005-T
Dougherty, E.R. and J. Astola, 1994. An Introduction to Nonlinear Image Processing. 1st Edn., SPIE Press, Bellingham, ISBN-10: 081941560X, pp: 182.

Gabbouj, M., 1996. Weighted Median Filtering-Striking Analogies to FIR Filters. In: Circuits and Systems Tutorials, Toumazou, C., N.C. Battersby and S. Porta (Eds.), John Wiley and Sons, New York, ISBN-10: 0780311701, pp: 5-21.

Kartik, A., S. Chandra, B. Ramamoorthy and S. Das, 1997. 3D tool wear measurement and visualisation using stereo imaging. Int. J. Mach. Tools Manuf., 37: 1573-1581. DOI: 10.1016/S0890-6955(97)00023-0

Luk, F., V. Hyunh and W. North, 1989. Measurement of surface roughness by a machine vision system. J. Phys. E, Sci. Instruments, 22: 977-980. DOI: 10.1088/0022-3735/22/12/001

Negoita, M.G., L. Sekanina and A. Stoica, 2008. Adaptive and evolvable hardware and systems: The state of the art and the prospectus for future development. Knowl. Based Intell. Inform. Eng. Syst., 5179: 310-318. DOI: 10.1007/978-3-54085567-5_39

Ramamoorthy, B. and V. Radhakrishnan, 1993. Statistical approaches to surface texture classification. Wear, 167: 155-161. DOI: 10.1016/0043-1648(93)90320-L

Samhouri, 2005. Surface roughness in grinding: offline identification with an adaptive neuro fuzzy inference system. Proceedings of the NAMRAC 33-2005 Conference, May 24-27, Columbia.

Tsai, D.M. and C.F. Tseng, 1999. Surface roughness classification for castings. Patt. Recog., 32: 389-405. DOI: 10.1016/S0031-3203(98)00077-6 